\title{
Late-onset startle syndrome and obsessive compulsive disorder
}

\author{
Alejandro Gonzalez and Michael P. Philpot* \\ Maudsley Hospital, London, UK
}

\begin{abstract}
A case of late onset sporadic startle syndrome in a patient with a right posterior fossa brain tumour is reported. The exaggerated startle response did not respond to treatment with clonazepam. In addition to anxiety and depression, the patient developed obsessive- compulsive symptoms which responded to behavioural therapy. The possible mechanisms for this unique pattern of symptoms are discussed.
\end{abstract}

Keywords: Startle, obsessive-compulsive, meningioma, aged

\section{Introduction}

The startle response is a normal primitive reaction which consists of a stereotyped pattern of movements including blinking, facial grimacing, flexion of the head, shoulders, elbows, trunk and knees. It may be elicited by any unexpected stimuli and will usually decrease in magnitude on repeated stimulation [2]. Response magnitude is influenced by individual factors such as personality [10], anxiety or fatigue [6], sleep or sensory deprivation [18] and the presence of a range of neurological and psychiatric conditions [8].

Startle 'disease' or hyperekplexia is an autosomal dominant condition in which the exaggerated startle response is a primary feature [2]. Major startle disease is also characterised by hypertonia in infancy, falling attacks without loss of consciousness, episodic generalised shaking, hyperreflexia and gait disturbance [10]. Minor startle disease consists only of an inconstant excessive startle response. Mutations of the $\alpha_{1}$ subunit of the glycine receptor (GLRA1) have been identified in effected pedigrees [3].

The patient we describe here, who came into our care at the age of $77 \mathrm{y}$, developed an exaggerated star-

${ }^{*}$ Corresponding author: Dr. Michael Philpot, Old Age Psychiatry Directorate, Maudsley Hospital, Denmark Hill, London SE5 8AZ, UK. Tel.: +44 171919 2193; Fax: +44 1719192171. tle response late in life in the presence of significant functional and organic mental features.

\section{Case description}

\subsection{Clinical history}

$\mathrm{K}$ was a right-handed retired shop-assistant without a previous psychiatric history. At the age of 67 she tripped and fell, dislocating her right shoulder. She became anxious and 3 months later suffered a myocardial infarction. Five days later she had a tonic-clonic seizure. Brain computed tomography (CT) revealed a calcified mass, probably a meningioma approximately $1.5 \mathrm{~cm}$ in diameter, arising from the inferior tentorium in right posterior fossa. There was no adjacent oedema but some displacement of the cerebellum. K had three more seizures during the following week but despite this $\mathrm{K}$ was fearful of surgical intervention which she refused. She was treated with phenytoin $300 \mathrm{mg} /$ day. The generalised seizures ceased but $\mathrm{K}$ continued to have 'blackouts' during which she would feel faint, experience parasthesiae in her right arm, fall slowly to the floor and jerk her limbs in an uncoordinated way. These attacks, which occurred about once a month until her death, would last up to two minutes, occurred only in the presence of others and were followed by rapid recovery. Although 'post-attack' prolactin levels were not taken her physicians at the time believed these were non-epileptic seizures or 'pseudo-seizures'. There was no evidence of cognitive impairment at this stage.

When $\mathrm{K}$ was $72 \mathrm{y}$ her husband died after a long illness. Shortly afterwards K became agitated and experienced panic attacks when shopping or travelling alone. Four months later she suddenly developed a weak dysphonic voice. ENT and speech therapy examinations revealed no organic abnormalities but K's symptoms persisted. A repeat brain CT showed no 
change in tumour size. At the age of $74 \mathrm{y}$, phenytoin was gradually withdrawn but over the following 4 weeks $\mathrm{K}$ developed a marked resting tremor and exaggerated startle response. The latter occurred in response to loud noises or bright lights and did not reduce in intensity on repeated stimulation. If sitting she would flex at the hips, shoulders and elbows and vigorously rub her hands against her temples, clench her eyes shut, look aghast and utter a worried cry. If standing she would suddenly fall to the floor with loss of muscle tone but without losing consciousness. The attacks were very brief, lasting only 10-15 seconds but severely disrupted her life.

At K's first psychiatric assessment (at $74.5 \mathrm{y}$ ) imipramine $75 \mathrm{mg} /$ day was prescribed for 'atypical' depression. Anxiety was reduced but K proved sensitive to anticholinergic side-effects at higher doses. Day hospital attendance and relaxation training led to a temporary reduction in agoraphobia. At age $75.5 \mathrm{y}$, the imipramine was changed to moclobemide rising to $600 \mathrm{mg} /$ day and flupenthixol $1 \mathrm{mg} /$ day. This lead to a partial remission of depression but 4 months later, when $\mathrm{K}$ was then $76 \mathrm{y}$, she developed obsessivecompulsive behaviour; checking lights, taps and locks, and washing her hands up to 12 times a day. Behavioural treatment helped but K's mood continued to fluctuate from day to day.

At 77 y K's startle response suddenly became more extreme and she began to stutter, a feature which worsened until her death. Clonazepam $4 \mathrm{mg} /$ day was substituted for flupenthixol. This had little effect on the startle response but did help her anxiety. Coincidently, K's accommodation was flooded and this precipitated another episode of compulsive washing and checking which again responded to behavioural therapy. Moclobemide had been unsuccessful in controlling the daily fluctuations in mood and was changed to clomipramine up to $75 \mathrm{mg}$ /day. This proved more successful in maintaining K's mood but at $77.5 \mathrm{y}$ she suffered a major stroke from which she did not regain consciousness. She died of bronchopneumonia 10 days later. An autopsy was not performed.

\subsection{Background history}

$\mathrm{K}$ was one of three children and was raised in care after being abandoned. Her father died in a psychiatric hospital at the age of 50, after 30 years of an unknown illness. She married at the age of 17 and had two children. A paternal aunt and one grandson suffered from epilepsy. $\mathrm{K}$ suffered from rheumatic fever at
$7 \mathrm{y}$ and had myocardial infarcts at 49 and $67 \mathrm{y}$. She suffered from angina and peripheral vascular disease for the last 30 years of her life. Although $\mathrm{K}$ was described as having been an anxious person she had not been unusually orderly or obsessional.

\subsection{Examination at age 77}

K was a small-framed, frail Caucasian woman. She was co-operative and made good eye contact. Interviews were repeatedly disrupted by her startle response. She spoke in a loud whisper and the flow of her speech was effected by her stutter. Although her mood varied, she persistently expressed a wish to die and often imagined suffocating herself. She was preoccupied with her physical health and the restrictions it placed upon her. She had insight into her condition and complied with treatment.

Cognitive performance fluctuated and depended on her mood. Concentration and recent memory were mildly impaired but orientation and remote memory were intact. She was unable to perform mental arithmetic including serial 3's but this was a life-long problem. There was no evidence of aphasia, apraxia or agnosia.

On neurological examination cranial nerves were normal. There was a cataract in the left eye and intermittent eyelid spasm. There was a resting tremor and she made frequent jerking movements of her upper limbs. Upper limb tone and power were normal but coordination and subjective sensation were bilaterally impaired. Lower limb tone was increased and power was slightly reduced. Coordination and sensation were within normal limits. Reflexes were unremarkable. Her gait was broad-based and she was unsteady on her feet. Examination of other systems revealed no abnormalities.

\subsection{Investigations}

Full blood count, ESR, liver, kidney and thyroid function, syphilis serology and serum folate were all normal. Vitamin B12 deficiency was discovered when $\mathrm{K}$ was $74 \mathrm{y}, 6$ months before the onset of her depression. Replacement therapy was commenced and subsequent serum B12 levels were satisfactory. Brain CT, first carried out when $\mathrm{K}$ was $67 \mathrm{y}$, was repeated every two years until her death. The tumour size remained unchanged in size over 10 years (see Fig. 1). An EEG performed at $75 \mathrm{y}$ showed a moderately high amplitude $11 \mathrm{~Hz}$ alpha rhythm in post-central regions which was 


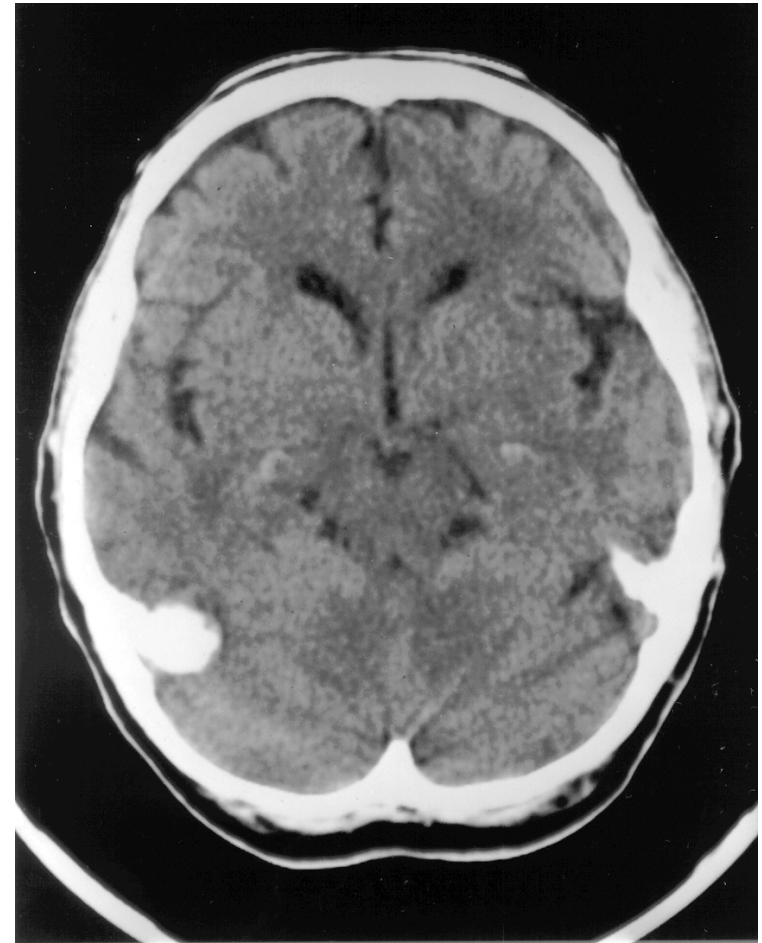

Fig. 1. Brain computed tomography performed when $\mathrm{K}$ was 74 years old. A right posterior fossa mass is shown.

responsive and symmetrical, some underlying theta components and diffuse low amplitude beta activity. However, the startle response to a provoking stimulus was not tested. Unfortunately she subsequently refused to have a further EEG.

\section{Discussion}

The physical and mental problems which $\mathrm{K}$ developed over a 10 year period may be summarised as follows: epilepsy and/or non-epileptic seizures, exaggerated startle response, dysphonia and stuttering, tremor, anxiety and depression, compulsive behaviour and mild cognitive impairment. Perhaps coincidentally she was discovered to have a right posterior fossa meningioma and vitamin B12 deficiency.

\subsection{Exaggerated startle}

Although the exaggerated startle response is reputedly a feature of many organic and functional psychiatric disorders [8], we can find no studies or case reports describing this problem in the elderly.

The meningioma may be of aetiological importance even though it did not change in size. Some displace- ment of the right cerebellum was reported in early scans. The intact cerebellar vermis is essential for the habituation of the startle response [11] and patients with minor startle disease have disrupted cerebellar function and fail to habituate to repeated stimuli [17].

Genetic factors are now thought to play a crucial role in startle disorders. Information on K's father's illness was not available but familial hyperekplexia cannot be excluded. The sporadic form is not linked to GLRA1 mutations and Andrews and Owen [3] suggest a number of reasons for this: a new mutation in the affected patient, non-penetrance in the parents or a phenocopy caused by organic disease. Apart from the late onset, $\mathrm{K}$ has the major features of sporadic startle disease [18]. Failure to respond to clonazepam has been reported in this disorder [13].

The association between excessive startle and sensitive personality or neurosis has been made $[7,16]$ but only cases with early onset are described. The relationship between startle and epilepsy is complex. In one case series of startle disease, $20 \%$ also suffered from epilepsy [13]. Conversely, the startle reaction may be so intense as to precipitate an epileptic seizure [1].

\subsection{Obsessive-compulsive disorder}

$\mathrm{K}$ developed obsessive-compulsive symptoms for the first time at $76 \mathrm{y}$. Obsessive-compulsive disorder is frequently co-morbid with major depression, although the precise degree of overlap in older people is not known [5]. In K's case, the compulsive symptoms developed, and later recurred, when her depressive symptoms were mild. The recurrence followed an adverse life event. Late onset of obsessive-compulsive disorder is rare and organic aetiologies have been postulated: an early history of rheumatic fever [15], reduced white matter volume in posterior cortical regions [9], right parietal infarction [14] and basal ganglia lesions [12] have all been implicated.

\section{Conclusion}

Calne et al. [4] have drawn attention to a number of neurological disorders in which subclinical neural damage early in life might combine with nerve cell loss as a result of ageing to give rise to symptoms much later in life. We would suggest that the apparently silent brain tumour via its effect on the cerebellum, in combination with risk factors including the family history of epilepsy and childhood rheumatic fever have combined to produce the unique constellation of symptoms described in this patient. 


\section{References}

[1] U. Aguglia, P. Tinuper and H. Gastaut, Startle-induced epileptic seizures, Epilepsy 25 (1984), 712-720.

[2] F. Andermann, D. Keene, E. Andermann and L.F. Quesney, Startle disease or hyperekplexia, Brain 103 (1980), 985-997.

[3] Andrews and Owen, Hyperekplexia: abnormal startle response due to glycine receptor mutations, British Journal of Psychiatry 170 (1997), 106-108.

[4] D. Calne, A. Eisen, E. McGeer and P. Spencer, Alzheimer's disease, Parkinson's disease, and motor neurone disease: abiotropic interaction between ageing and environment?, Lancet ii (1986), 1067-1070.

[5] M.R. Eastwood and J. Lindesay, Epidemiology, in: Neurotic Disorders in the Elderly, J. Lindesay, ed., Oxford Medical Publications, Oxford, 1995, pp. ??-??.

[6] P. Eckman, W. Friesan and R.C. Simons, Is the startle reaction an emotion?, Journal of Personality and Social Psychology 49 (1985), 1416-1426.

[7] J. Hardison, Are the jumping Frenchman of Maine goosey?, Journal of the American Medical Association 244 (1980), 70.

[8] R. Howard and R. Ford, From the jumping Frenchmen of Maine to post-traumatic stress disorder: the startle response in neuropsychiatry, Psychological Medicine 22 (1992), 695707.

[9] M.A. Jenike, H.C. Breiter, L. Baer, D.N. Kennedy, C.R. Savage, M.J. Olivares, R.L. O'Sullivan, D.M. Shera, S.L. Rauch, N. Kreuthen, B.R. Rosen, V.S. Caviness, P.A. Filipek, Cere- bral structural abnormalities in obsessive-compulsive disorder, Archives of General Psychiatry 53 (1996), 625-632.

[10] T. Kurczynski, Hyperekplexia, Archives of Neurology 40 (1983), 246-248.

[11] R.N. Leaton and W.F. Supple, Cerebellar vermis: essential for long-term habituation of the acoustic startle response, Science 232 (1986), 513-515.

[12] F. Lopez-Rodriguez, I. Gunay and N. Glaser, Obsessive compulsive disorder in a woman with left basal ganglia infarct: a case report, Behavioural Neurology 10 (1997), 101-103.

[13] E. Saenz-Lope, F.J. Herranz-Tannaro, J.C. Masdeu and J.R. Pena, Hyperekplexia: a syndrome of pathological startle response, Annals of Neurology 15 (1984), 36-41.

[14] S. Simpson and B. Baldwin, Neuropsychiatry and SPECT of an acute obsessive-compulsive syndrome patient, British Journal of Psychiatry 166 (1995), 390-392.

[15] S.E. Swedo, J.L. Rapoport, D.L. Cheslow, H.L. Leonard, E.M. Ayoub, D.M. Hosier, E.R. Wald, High prevalence of obsessive compulsive symptoms in patients with Sydenham's chorea, American Journal of Psychiatry 146 (1989), 246255.

[16] F.C. Thorne, Startle neurosis, American Journal of Psychiatry 101 (1994), 105-109.

[17] M.A.J. Tijssen, G.W. Padberg and G. van Dijk, The startle pattern in the minor form of hyperekplexia, Archives of Neurology 53 (1996), 608-613.

[18] E. Wilkins, M. Hallet and M. Wess, Audiogenic startle reflex of man and its relationship to startle syndromes, Brain 109 (1986), 561-573. 


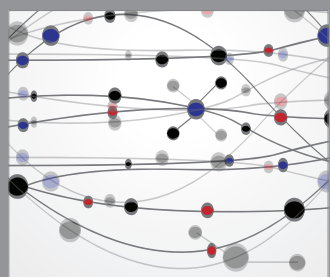

The Scientific World Journal
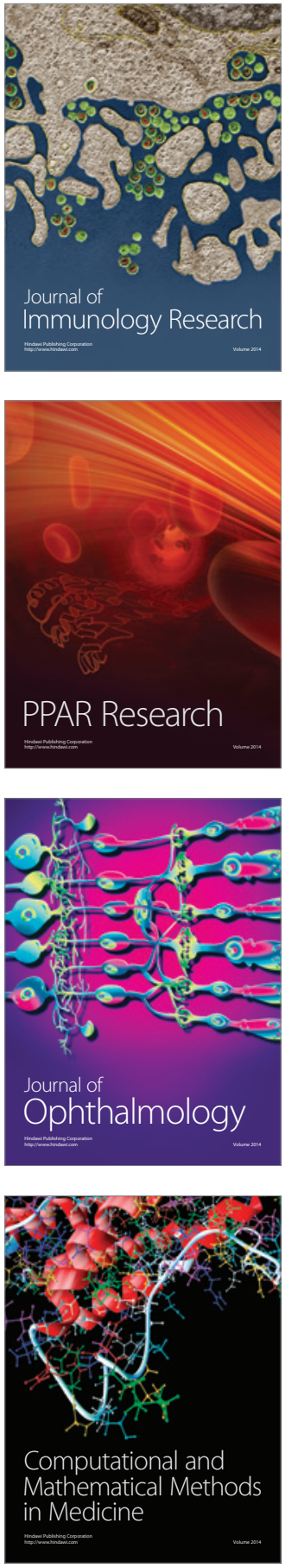

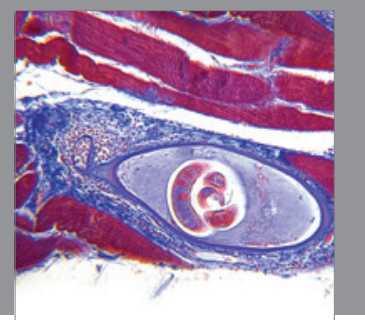

Gastroenterology

Research and Practice
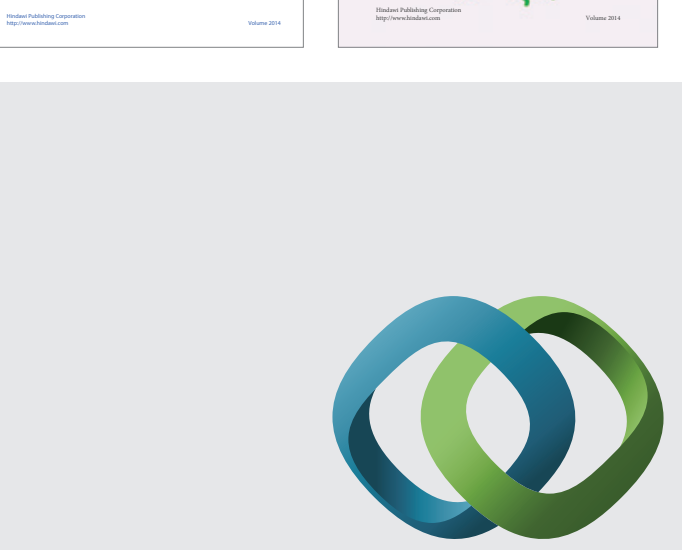

\section{Hindawi}

Submit your manuscripts at

http://www.hindawi.com
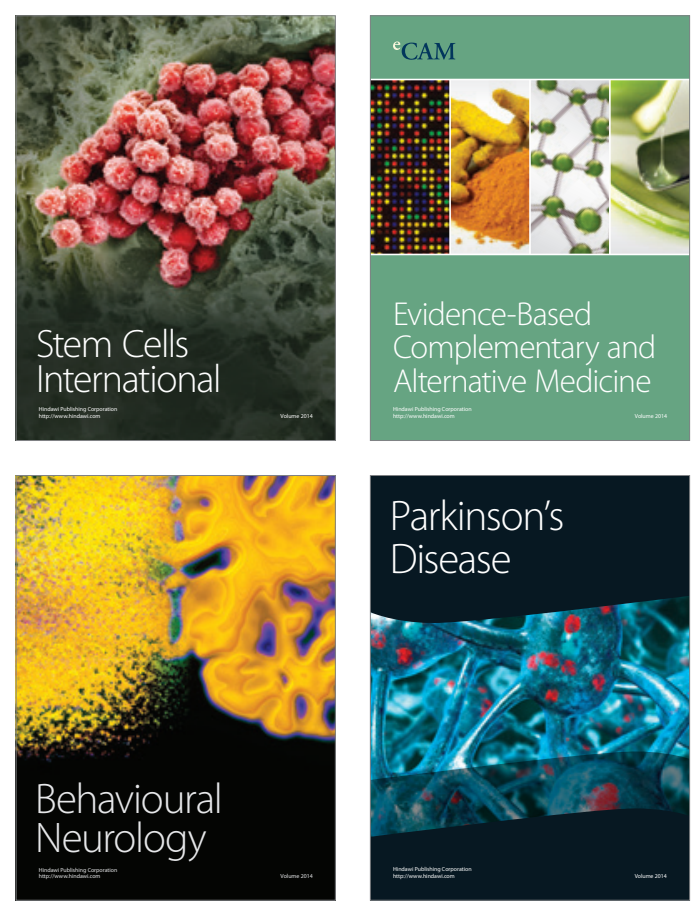

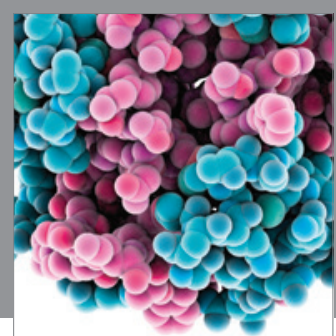

Journal of
Diabetes Research

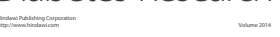

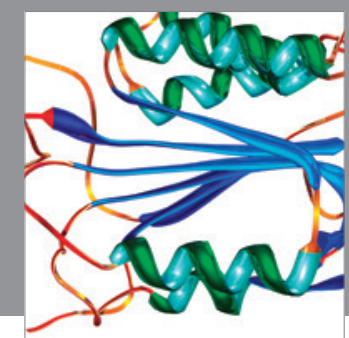

Disease Markers
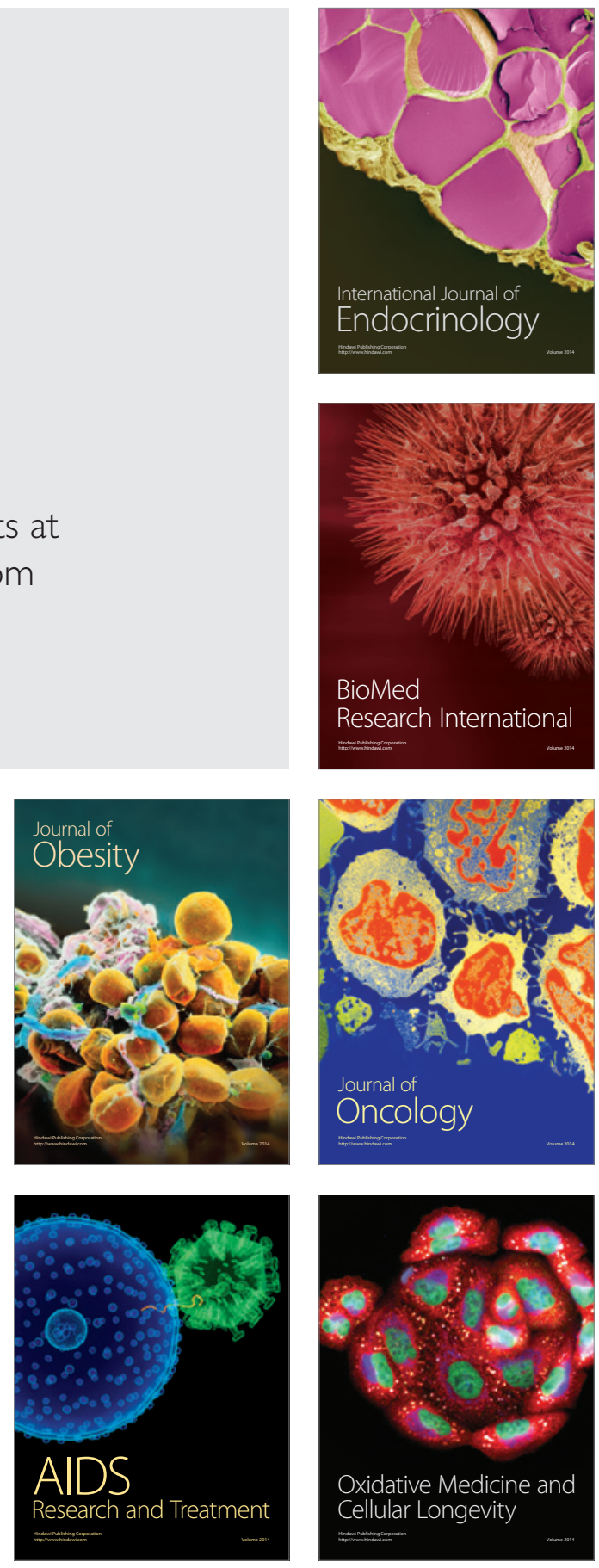